\title{
Adaptive curvelet-domain primary-multiple separation
}

\author{
Felix J. Herrmann ${ }^{1}$, Deli Wang ${ }^{2}$, and Dirk J. (Eric) Verschuur ${ }^{3}$
}

\begin{abstract}
In many exploration areas, successful separation of primaries and multiples greatly determines the quality of seismic imaging. Despite major advances made by surface-related multiple elimination (SRME), amplitude errors in the predicted multiples remain a problem. When these errors vary for each type of multiple in different ways (as a function of offset, time, and dip), they pose a serious challenge for conventional least-squares matching and for the recently introduced separation by curvelet-domain thresholding. We propose a data-adaptive method that corrects amplitude errors, which vary smoothly as a function of location, scale (frequency band), and angle. With this method, the amplitudes can be corrected by an elementwise curvelet-domain scaling of the predicted multiples. We show that this scaling leads to successful estimation of primaries, despite amplitude, sign, timing, and phase errors in the predicted multiples. Our results on synthetic and real data show distinct improvements over conventional least-squares matching in terms of better suppression of multiple energy and high-frequency clutter and better recovery of estimated primaries.
\end{abstract}

\section{INTRODUCTION}

Surface-related multiple elimination (SRME) (Verschuur et al., 1992; Fokkema and van den Berg, 1993; Berkhout and Verschuur, 1997; Weglein et al., 1997) involves two stages, multiple prediction and primary-multiple separation. During the second stage, measures are taken to compensate for imperfections in the multiple predictions. For SRME, predicted multiples often include source signatures and directivity patterns that differ from those present in the data (see, e.g., Verschuur et al., 1992; Ikelle et al., 1997). Moreover, 2D SRME produces errors in the predicted multiples because of 3D complexity of the earth (Dragoset and Jeričević, 1998; Ross et al., 1999; Verschuur, 2006), whereas recently developed full 3D-SRME algorithms can suffer from imperfections related to incomplete acquisitions (see, e.g., Lin et al., 2004; Moore and Dragoset, 2004; van Borselen et al., 2004; van Dedem and Verschuur, 2005), including erroneous reconstructions of missing near offsets (Dragoset and Jeričević, 1998). For field data, these factors preclude iterative SRME, resulting in amplitude errors that vary for different multiple orders (see, e.g., Verschuur and Berkhout, 1997; Paffenholz et al., 2002).

In practice, the second separation stage appears to be particularly challenging because adaptive $\ell_{2}$-matched-filtering techniques are known to lead to residual multiple energy, high-frequency clutter, and deterioration of the primaries (Chen et al., 2004; Abma et al., 2005; Herrmann et al., 2007a). By employing the ability of the curvelet transform (Candes et al., 2006; Hennenfent and Herrmann, 2006) to detect wavefronts with conflicting dips (e.g., caustics), Herrmann et al. (2007a) and Herrmann et al. (2008b) derived a nonadaptive separation scheme (independent of the total data) that uses the original data and SRME-predicted multiples as input and produces an estimate for the primaries. This threshold-based method proved to be robust with respect to moderate errors (sign, phase, and timing) in the predicted multiples and derived its success from the sparsifying property of curvelets for data with wavefronts. Despite recent advances in thresholding by a Bayesian formulation (Saab et al., 2007; Wang et al., 2007) and mitigation of the effects of missing data (Hennenfent and Herrmann, 2008; Herrmann et al., 2007b), curvelet-domain separation deteriorates when predicted multiples have significant amplitude errors. Thresholding in these cases can give rise to inadvertent removal of primary energy or to remnant multiple energy.

Manuscript received by the Editor 29 August 2007; revised manuscript received 5 November 2007; published online 2 May 2008.

${ }^{1}$ University of British Columbia, Department of Earth and Ocean Sciences, Seismic Laboratory for Imaging and Modeling, Vancouver, Canada. E-mail: fherrmann@eos.ubc.ca.

${ }^{2}$ Jilin University, College of Geoexploration Science and Technology, Changchun, China. Visiting the University of British Columbia. E-mail: wangdeli@ email.jlu.edu.cn.

${ }^{3}$ Delft University of Technology, Department of Applied Physics, Laboratory of Acoustical Imaging and Sound Control. E-mail: d.j.verschuur@ @tg.tudelft.nl. (c) 2008 Society of Exploration Geophysicists. All rights reserved. 


\section{Our contribution}

We present a new technique that mitigates the effects of unbalanced amplitudes that vary relatively smoothly along the locations and dips of predicted multiples. Our approach is complementary to windowed matched-filtering techniques (Verschuur and Berkhout, 1997), which offer limited control over window-to-window variations among the estimated matched filters. Our method also avoids the relatively expensive multiple predictions required by iterative SRME. To offer better control over these variations, errors in the single-window SRME-predicted multiples are modeled by a zero-order pseudodifferential operator, a kind of spatially varying dip filter, which can be approximated well by diagonal curvelet-domain scaling (Herrmann et al., 2008a). This scaling is estimated from the input data and predicted multiples by a nonlinear optimization procedure, during which smoothness among neighboring curvelet coefficients is imposed. This smoothness among the curvelet coefficients ensures a scaling that is well behaved spatially and as a function of dip. This approach employs the adaptability of curvelets and the smoothness constraint prevents overfitting of the data, which can lead to a loss of primary energy. Although distinct, our approach is similar to recent work in migration-amplitude recovery, in which scaling methods with smoothness constraints have been proposed (Guitton, 2004; Symes, 2008). This paper builds explicitly on a curvelet-based approach to this problem introduced by Herrmann et al. (2008a).

\section{THEORY}

The proposed separation method consists of two stages. During the first adaptive stage, the predicted multiples $\check{\mathbf{s}}_{2}$ are fitted through a correction operator to the multiples present in the total data, $\mathbf{p}=\mathbf{s}_{1}$ $+\mathbf{s}_{2}$, which consists of the sum of primaries $\mathbf{s}_{1}$ and multiples $\mathbf{s}_{2}$. During the second stage, the primaries and multiples are separated by a thresholding procedure, defined in terms of the scaled magnitudes of the curvelet coefficients of the predicted multiples. Because SRMEpredicted multiples are used as input, the wavelet and source directivity will not be compensated properly (Verschuur et al., 1992).

\section{The forward model}

Without the wavelet and source directivity, the predicted multiples can be regarded as a scaled version(along the time and receiver or offset axes) of the true multiples. Mathematically, this nonstationary "scaling" can be represented by a pseudodifferential operator. For our application, this operator acts on shot records or on commonoffset panels and applies a location, frequency, and dip-dependent zero-phase scaling. By applying a matrix-vector multiplication to predicted multiples, this operator models the true multiples in the data, i.e.,

$$
\mathbf{s}_{2}=\mathbf{B} \check{\mathbf{s}}_{2},
$$

where $\mathbf{B}$ is a full positive-definite matrix, implementing the action of the pseudodifferential operator, and $\check{\mathbf{s}}_{2}$ represents the predicted multiples, calculated with single-windowed convolutional matched filtering. Relating the predicted multiples to true multiples offers flexibility to model amplitude mismatches. Note, however, that this model cannot incorporate kinematic shifts because pseudodifferential operators are unable to move wavefronts.

By compensating for the source wavelet and directivity via a conventional local matched-filtering procedure, the pseudodifferential operator becomes zero order and permits a diagonal curvelet-domain decomposition (Herrmann et al., 2008a),

$$
\mathbf{s}_{2} \approx \mathbf{C}^{T} \operatorname{diag}\{\mathbf{w}\} \check{C}_{2}, \quad\{w\}_{\mu \in \mathbf{M}}>0,
$$

with $\mathbf{C}$ the 2D discrete curvelet transform (see, e.g., Candes et al., 2006; Hennenfent and Herrmann, 2006), w the curvelet-domain scaling vector, and $\mathbf{M}$ the index set of curvelet coefficients. Because we are using the curvelet transform based on wrapping, which is a tight frame, $\mathbf{C}^{T} \mathbf{C}=\mathbf{I}$, and the transpose, denoted by the symbol $T$, equals the pseudoinverse.

In this approximate forward model, for which precise theoretical error estimates exist (Herrmann et al., 2008a), the predicted multiples are linked to the actual multiples by a simple curvelet-domain scaling. This curvelet-domain scaling applies a location, scale, and dip-dependent amplitude correction. Because the matrix $\mathbf{B}$ is positive-definite, the entries in the scaling vector $\mathbf{w}$ are positive. This approximate formulation of the forward model forms the basis for our curvelet-domain matched filter.

\section{Curvelet-domain matched filtering}

Equation 2 lends itself to an inversion for the unknown scaling vector. Because the true multiples are unknown, our formulation minimizes the least-squares mismatch between the total data and the predicted multiples. The following issues complicate the estimation of the scaling vector: (1) the undeterminedness of the forward model, resulting from redundancy of the curvelet transform (i.e., $\mathbf{C C}^{T}$ ), is rank deficient; (2) there is a risk of overfitting the data, which leads to unwanted removal of primary energy; and (3) there is a positivity requirement for the scaling vector. To address issues 1 and 2, the following augmented system of equations is formed which relates the unknown scaling vector $\mathbf{w}$ to the augmented data vector $\mathbf{d}$, i.e.,

$$
\left[\begin{array}{l}
\mathbf{p} \\
\mathbf{0}
\end{array}\right]=\left[\begin{array}{c}
\mathbf{C}^{T} \operatorname{diag}\left\{\check{C \check{s}}_{2}\right\} \\
\gamma \mathbf{L}
\end{array}\right] \mathbf{w}
$$

or $\mathrm{d}=\mathbf{F}_{\gamma} \mathbf{w}$. The scaling vector is found by minimizing the functional

$$
J_{\gamma}(\mathbf{z})=\frac{1}{2}\left\|\mathbf{d}-\mathbf{F}_{\gamma} e^{\mathbf{z}}\right\|_{2}^{2}
$$

where the substitution of $\mathbf{w}=e^{\mathrm{z}}$ (with the exponentiation taken elementwise) guarantees positivity (issue 3) of the solution (Vogel, 2002). This formulation seeks a solution fitting the total data with a smoothness constraint imposed by the sharpening operator $\mathbf{L}$, which for each scale penalizes fluctuations among neighboring curvelet coefficients in the space and angle directions (see Herrmann et al., 2008a, for a detailed description). The amount of smoothing is controlled by the parameter $\gamma$. For increasing $\gamma$, there is more emphasis on smoothness at the expense of overfitting the data (i.e., erroneously fitting the primaries). For a specific $\gamma$, the penalty functional in equation 4 is minimized with respect to the vector $\mathbf{z}$ with the limitedmemory BFGS (Nocedal and Wright, 1999) with the gradient

$$
\operatorname{grad} J(\mathbf{z})=\operatorname{diag}\left\{e^{\mathbf{z}}\right\}\left[\mathbf{F}_{\gamma}^{T}\left(\mathbf{F}_{\gamma} e^{\mathbf{z}}-\mathbf{d}\right)\right] .
$$

Ideally, the solution of the above optimization problem, $\widetilde{\mathbf{z}}$ $=\arg \min _{\mathbf{z}} J(\mathbf{z})$, would yield, after application of the data-dependent scaling, the appropriate prediction for the multiples. Unfortunately, other phase and kinematic errors might interfere, rendering a separation based on the residual alone (as in SRME) ineffective (i.e., 
$\mathbf{p}-\mathbf{C}^{T} \operatorname{diag}\{\widetilde{\mathbf{w}}\} \mathbf{C} \check{\mathbf{s}}_{2}$ with $\widetilde{\mathbf{w}}=e^{\tilde{\mathbf{z}}}$ is an inaccurate estimate for the primaries). Robustness of threshold-based primary-multiple separation addresses this important issue and forms the second nonadaptive stage of our separation scheme.

\section{Primary-multiple separation by curvelet-domain thresholding}

Because of the curvelet's sparsity and parameterization (by position, scale, and dip) primaries and multiples naturally separate in this domain. This property explains the success of threshold-based primary-multiple separation. According to the latest development in threshold-based primary-multiple separation (Saab et al., 2007; Wang et al., 2007), the estimated primaries are given by

$$
\check{\mathbf{s}}_{1}=\operatorname{Bayes}(\mathbf{p}, \mathbf{t}),
$$

with the operator Bayes $(\cdot, \cdot)$ denoting primary estimation by our iterative Bayesian separation scheme (detailed in Saab et al., 2007), which uses the total data and a curvelet-domain threshold vector $\mathbf{t}$ as input and which produces the estimated primaries $\widetilde{\mathbf{S}}_{1}$. This curveletdomain threshold is given by the absolute values of the (scaled) predicted multiples. Equation 6 is an instance of a nonadaptive curveletdomain primary-multiple procedure which, as reported in the literature (Herrmann et al., 2007a; Herrmann et al., 2008b; Saab et al., 2007; Wang et al., 2007), has been applied successfully been to synthetic- and real-data examples.

\section{APPLICATION}

We test the above-described adaptive separation algorithm by examining synthetic and real data. The main purpose of these tests is to study the improvement by curvelet-domain matching compared with optimized results for single-iteration SRME. This case is relevant for situations in which data quality does not permit iterative SRME or the cost of multiple iterations of SRME is a concern. In either situation, the predicted multiples will contain amplitude errors that might give rise to residual multiple energy and dimmed primaries. We show that the proposed scaling by curvelet-domain matched filtering improves the estimation for primaries as long as the curvelet-to-curvelet variations for this scaling are controlled sufficiently by the smoothness constraint. Relaxation of this constraint can lead to overfitting and hence to inadvertent removal of primary energy.

\section{Synthetic-data example}

We consider a shot record from a synthetic line, generated by an acoustic finite-difference code for a velocity model that consists of a high-velocity layer, which represents salt, surrounded by sedimentary layers, and a water bottom that is not completely flat (see Figure 11 in Herrmann et al., 2007a). In Figure 1, the results for optimized single-term SRME are compared with curvelet-domain Bayesian separation with and without our amplitude scaling. Figure 1a-c includes the total input data with multiples, the SRME-predicted multiples, and the multiple-free data, respectively. The predicted multiples are the result of conventional matching in a single window. The multiple-free data were modeled with an absorbing boundary condition, removing the surface-related multiples. Results for the estimated primaries according to optimized single-term SRME with windowed matching, Bayesian separation, and scaled-Bayesian separation are included in Figure 1d-f. Comparison of these results shows a significant improvement for the primaries computed with the curvelet-domain amplitude scaling, calculated by solving equation 4 for $\gamma=0.5$. For this choice of $\gamma$, the multiples are not overfitted, and the amplitude correction leads to a removal of remnant multiple energy, particularly for the events annotated by arrows. The value for $\gamma$ was found experimentally. Finally, note that the improvement in the a)

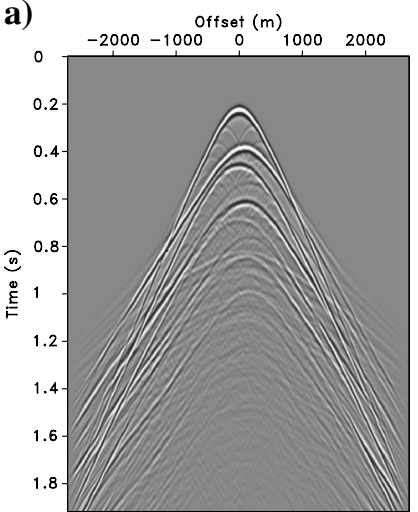

c)

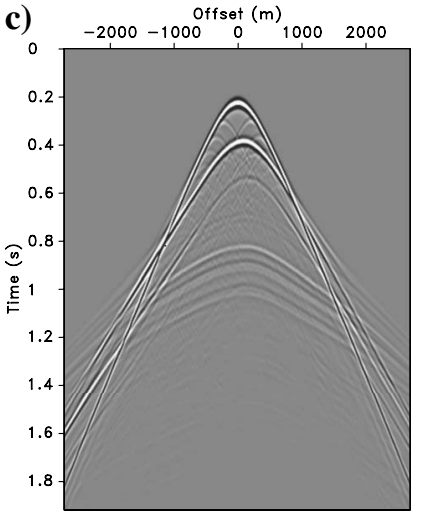

e)

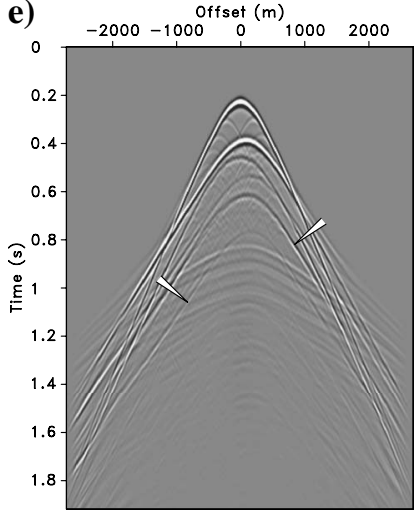

b)

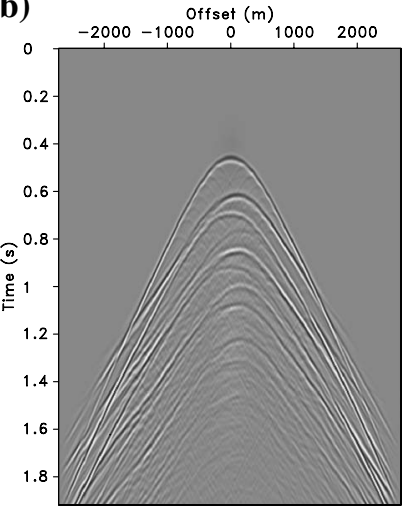

d)

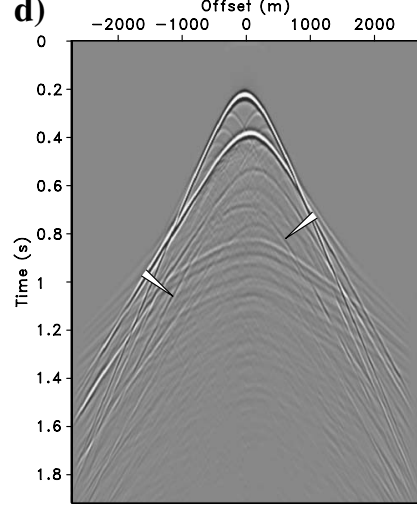

f)

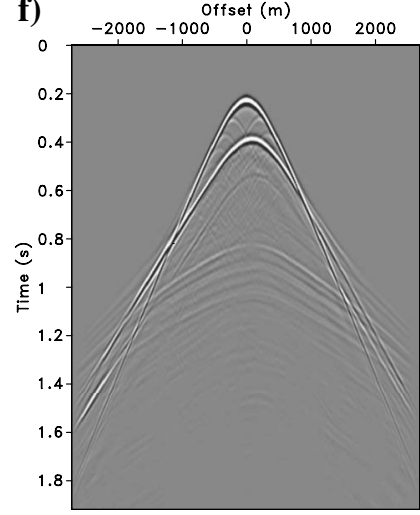

Figure 1. Primary-multiple separation on a synthetic shot record. (a) Total data $\mathbf{p}$, including primaries and multiples. (b) Single-term SRME-predicted multiples wavelet-matched within a global window $\left(\check{\mathbf{s}}_{2}\right)$. (c) Reference surface-related multiple-free data modeled with an absorbing boundary condition. (d) Estimate for primaries, yielded by optimized one-term SRME computed with a windowedmatched filter. (e) Estimate for primaries, computed by Bayesian iterative thresholding with a threshold defined by $t=\left|\mathbf{C s}_{2}\right|$. (f) The same as (e) but for the scaled threshold, i.e., $t=\left|\operatorname{diag}\{\widetilde{\mathbf{w}}\} \mathbf{C} \check{\mathbf{s}}_{2}\right|$ (with $\gamma=0.5$ ). Notice the improvement for the scaled estimate for primaries, compared with primaries yielded by SRME in (d) and by the Bayesian separation without scaling in (e). 
estimate for primaries results from the combination of curvelet-domain separation and scaling, yielding results comparable to those expected from multiterm SRME. Even though multiterm SRME, in combination with standard $\ell_{2}$-subtraction, is known to remove surface-related multiples for synthetic data nearly perfectly, SRME in practice is often viable for only one iteration because field data sets often do not obey assumptions of the model. Therefore, the singleterm SRME result in Figure 1d can be considered as state of the art.

\section{Real-data example}

Figure 2a contains the common-offset section (at an offset of $200 \mathrm{~m}$ ) that we selected from a North Sea field data set. Estimated primaries according to conventional SRME are plotted in Figure $2 b$. Results in which $\ell_{2}$-matched filtering in the shot domain (Verschuur and Berkhout, 1997) is replaced by Bayesian thresholding (Saab et al., 2007) in the offset domain are presented for a single offset in Figure $2 \mathrm{c}$ without scaling and in Figure $2 \mathrm{~d}$ with scaling. The scaled result is calculated for $\gamma=0.3$. Juxtaposing the standard SRME and the curvelet-based results shows a removal of high-frequency clutter, in agreement with earlier findings reported in the literature. Moreover, primaries in the deeper part of the section (e.g., near the
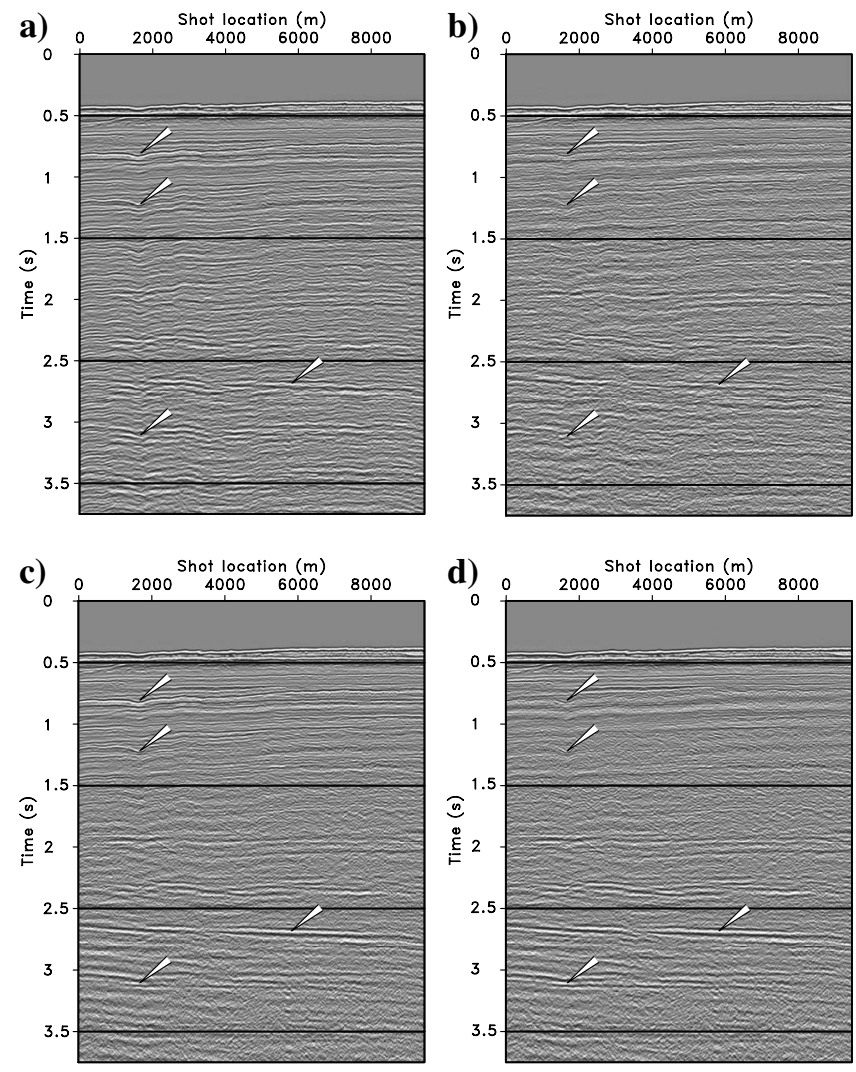

Figure 2. Adaptive curvelet-domain primary-multiple separation on real data. (a) Near-offset (200-m) section for total data plotted with automatic gain control. (b) Estimate for the primaries, yielded by optimized one-term SRME computed with a windowed-matched filter. (c) Estimate for primaries, computed by Bayesian iterative thresholding with a threshold defined by $t=\left|\mathbf{C} \check{\mathbf{s}}_{2}\right|$. (d) The same as (c) but for the scaled (for $\gamma=0.3$ ) threshold, i.e., $\mathbf{t}=\left|\operatorname{diag}\{\widetilde{\mathbf{w}}\} \mathbf{C} \check{\mathbf{s}}_{2}\right|$. Notice the improvement for the scaled estimate for primaries, compared with the primaries yielded by SRME in (b) and by the Bayesian separation without scaling in (c). two lower arrows in each plot) are preserved much better compared with the standard SRME result. Removal of strong residual multiples in the shallow part (e.g., first- and second-order water bottom multiples indicated by the arrows around 0.75 and $1.20 \mathrm{~s}$ ) is particularly exciting. Because of the unbalanced amplitudes of the predicted multiples, neither standard SRME nor nonadaptive Bayesian thresholding can eliminate these events. Our adaptive method, however, successfully removes these events through use of curvelet-domain scaling. Compared with nonadaptive thresholding, residual multiples are better suppressed, whereas our adaptive scheme also leads to at least similar but often even better overall continuity and amplitude preservation of the estimated primaries. For example, improvements are visible in the lower left corner of the sections (between offsets 0 and $2000 \mathrm{~m}$ and times 3.0 and $3.6 \mathrm{~s}$ ), where lowfrequency multiple residuals are better suppressed after curvelet-domain matched filtering ( $\mathrm{cf}$. Figure $2 \mathrm{c}$ and $\mathrm{d}$ ) without deterioration of the primary energy. Finally, observe the improved recovery of primary energy at the lower arrow in Figure 2d, compared with the primary in Figure 2c.

\section{CONCLUSIONS}

We present a method that improves estimates for primaries for situations in which multiterm SRME is nonviable. Our alternative augments Bayesian primary-multiple separation with a data-adaptive step during which amplitudes of the predicted multiples are matched to multiples in the data. This match is achieved in the curvelet domain, which allows for position, scale, and dip-dependent amplitude correction through diagonal scaling of the transform coefficients. Overfitting (i.e., distortion of the primaries) during the matching is avoided by promoting smoothness among neighboring coefficients in the scaling vector. Application of our method to synthetic and real data sets shows a clear improvement in multiple suppression and primary preservation, which can be attributed to the curvelet-domain amplitude correction by scaling. Because our correction is based on a relatively mild smoothness assumption, stating that the amplitude errors cannot vary too rapidly as a function of position, scale, and angle, we envisage applications in other areas, such as suppression of internal multiples, in which angle-dependent reflection and transmission errors play a role.

\section{ACKNOWLEDGMENTS}

The authors would like to thank R. Saab and Ö. Yilmaz for developing the Bayesian thresholding technique and C. C. Stolk for his implementation of the curvelet-domain smoothness constraint. We also would like to thank the authors of CurveLab (www.curvelet.org). The plots were prepared with Madagascar (rsf.sf.net). The matched-filter method was implemented in scientific python (www.scipy.org) with PyCurveLab (https://wave.eos.ubc.ca/Software/ Licenced/). We thank Norsk Hydro for the field data set. This work was supported financially in part by NSERC Discovery (22R81254) and CRD grants DNOISE (334810-05) of F. J. H. and was carried out as part of the SINBAD project with support, secured through ITF, from BG Group, BP, Chevron, ExxonMobil, and Shell. 


\section{REFERENCES}

Abma, R., N. Kabir, K. H. Matson, S. Michell, S. A. Shaw, and B. McLain, 2005, Comparisons of adaptive subtraction methods for multiple attenuation: The Leading Edge, 24, 277-280.

Berkhout, A. J., and D. J. Verschuur, 1997, Estimation of multiple scattering by iterative inversion, Part I: Theoretical considerations: Geophysics, 62 $1586-1595$.

Candes, E. J., L. Demanet, D. L. Donoho, and L. Ying, 2006, Fast discrete curvelet transforms: Multiscale Modeling and Simulation, 5, 861-899.

Chen, J., E. Baysal, and Ö. Yilmaz, 2004, Surface related multiple attenuation on the Sigsbee2B dataset: 74th Annual International Meeting, SEG, Expanded Abstracts, 1329-1332.

Dragoset, W. H., and Z. Jeričević, 1998, Some remarks on multiple attenuation: Geophysics, 63, 772-789.

Fokkema, J. T., and P. M. van den Berg, 1993, Seismic applications of acoustic reciprocity: Elsevier.

Guitton, A., 2004, Amplitude and kinematic corrections of migrated images for nonunitary imaging operators: Geophysics, 69, 1017-1024.

Hennenfent, G., and F. J. Herrmann, 2006, Seismic denoising with non-uniformly sampled curvelets: IEEE Computing in Science and Engineering, 8, 16-25.

, 2008, Simply denoise: Wavefield reconstruction via coarse nonuniform sampling: Geophysics, this issue.

Herrmann, F. J., U. Boeniger, and D. J. Verschuur, 2007a, Nonlinear primarymultiple separation with directional curvelet frames: Geophysical Journal International, 170, 781-799.

Herrmann, F. J., P. P. Moghaddam, and C. C. Stolk, 2008a, Sparsity- and continuity-promoting seismic imaging with curvelet frames: Applied and Computational Harmonic Analysis, 24, no. 2, Special issue, Mathematical Imaging, part II, 150-173.

Herrmann, F. J., D. Wang, and G. Hennenfent, 2007b, Multiple prediction from incomplete data with the focused curvelet transform: 77 th Annual International Meeting, SEG, Expanded Abstracts, 2505-2509.

Herrmann, F. J., D. Wang, G. Hennenfent, and P. Moghaddam, 2008b, Curvelet-based seismic data processing: a multiscale and nonlinear approach: Geophysics, 73, no. 1, A1-A5.
Ikelle, L., G. Roberts, and A. Weglein, 1997, Source signature estimation based on the removal of first-order multiples: Geophysics, 62, 1904-1920.

Lin, D., J. Young, Y. Huang, and M. Hartmann, 2004, 3D SRME application in the Gulf of Mexico: 74th Annual International Meeting, SEG, Expanded Abstracts, 1257-1260.

Moore, I., and W. Dragoset, 2004, 3-D surface-related multiple prediction (SMP): 74th Annual International Meeting, SEG, Expanded Abstracts, 1249-1252.

Nocedal, J., and S. J. Wright, 1999, Numerical optimization: Springer.

Paffenholz, J., B. McLain, and P. Keliher, 2002, Subsalt multiple attenuation and imaging: Observations from the Sigsbee2B synthetic dataset: $72 \mathrm{nd}$ Annual International Meeting, SEG, Expanded Abstracts, 2122-2125.

Ross, W. S., Y. Yu, and F. A. Gasparotto, 1999, Traveltime prediction and suppression of 3-D multiples: Geophysics, 64, 261-277.

Saab, R., D. Wang, Ö. Yilmaz, and F. J. Herrmann, 2007, Curvelet-based primary-multiple separation from a Bayesian perspective: 77th Annual International Meeting, SEG, Expanded Abstracts, 2510-2514.

Symes, W. W., 2008, Approximate linearized inversion by optimal scaling for prestack depth migration: Geophysics, 73, no. 2, R23-R35.

van Borselen, R., R. Schonewille, and R. Hegge, 2004, 3-D SRME: Acquisition and processing solutions: 74th Annual International Meeting, SEG, Expanded Abstracts, 1241-1244.

van Dedem, E. J., and D. J. Verschuur, 2005, 3-D surface-related multiple prediction: A sparse inversion approach: Geophysics, 70, V31-V43.

Verschuur, D. J., 2006, Seismic multiple removal techniques: Past, present and future: EAGE Publications BV.

Verschuur, D. J., and A. J. Berkhout, 1997, Estimation of multiple scattering by iterative inversion, Part II: Practical aspects and examples: Geophysics, 62, 1596-1611.

Verschuur, D. J., A. J. Berkhout, and C. P. A. Wapenaar, 1992, Adaptive surface-related multiple elimination: Geophysics, 57, 1166-1177.

Vogel, C., 2002, Computational methods for inverse problems: SIAM.

Wang, D., R. Saab, O. Yilmaz, and F. J. Herrmann, 2007, Recent results in curvelet-based primary-multiple separation: Application to real data: 77 th Annual International Meeting, SEG, Expanded Abstracts, 2500-2504.

Weglein, A. B., F. A. Carvalho, and P. M. Stolt, 1997, An inverse scattering series method for attenuating multiples in seismic reflection data: Geophysics, 62, 1975-1989. 